\title{
IN SITU STUDY OF THE MARTENSITIC TRANSFORMATION OF Cu-Zn-Al SHAPE MEMORY ALLOY SINGLE CRYSTALS
}

\author{
C. JOURDAN, J. GASTALDI, G. GRANGE, G. GUÉNIN* and S. BELKAHLA*,(1) \\ CRMC2-CNRS, Campus de Luminy, case 913, F-13288 Marseille cedex 9, France \\ *Institut National des Sciences Appliquées de Lyon, Groupe de Métallurgie Physique et de Physique \\ des Matériaux, ERA CNRS $N^{\circ} 341$, Bât. 502, 20 avenue A. Einstein, F-69621 Villeurbanne, France
}

\begin{abstract}
We have studied by synchrotron ${ }^{\#}$ white beam X-Ray Topography , in situ and in real time, the thermoelastic transformation of the $\mathrm{Cu}-\mathrm{Zn}-\mathrm{Al}$ shape memory alloy. The evolution of the first martensite lath has been followed during the temperature variation and its interaction with the crystalline defects of the parent phase has been shown.
\end{abstract}

\section{1.- Introduction}

Some electron microscopic studies have been previously carried out on $\mathrm{B} \mathrm{Cu-Zn-Al} \mathrm{shape}$ memory alloys in order to determine either the cristallographic relations between the two phases (1) or the crystalline defects role in the thermoelastic transformation (2). The results brought by this technique are interesting but they are incomplete and they do not account for the bulk material comportement. The long range strains associate with the transformation of shape memory alloys are not displayed.

This thermoelastic transformation has already been studied by X-Ray topography with conventional X-Ray generators (3). Two difficulties have made impossible this in situ study of the transformation : a very long exposure time ( $>24$ hours) due to the low energy of the X-Ray beam and the impossibility, resulting from the monochromaticity of the XRay beam, to record the diffracted beam of the low temperature phase.

The in situ and real time Synchrotron White Beam X-Ray Topography (SWBXRT) technique is well adapted to the study of the evolution processes of bulk crystalline materials submitted to various treatments. Its possibilities were successfully used in several studies : recrystallization (4), growth from the liquid phase (5) and solid-solid phase transition (6).

In this paper we present the SWBXRT study of the thermoelastic transformation of the $\mathrm{Cu}-\mathrm{Zn}$-Al shape memory alloy. The high energy beam reduces the exposure time to the second and the white beam allows to record simultaneously the diffraction diagram of the high and low temperature phases.

(\#) L.U.R.E., 91405 Orsay cedex, France

(1) On leave of absence from University of Annaba, Institut of Physics,

BP. 12, Annaba, Algeria 


\section{2.- Experiments}

The appropriate alloy composition $\mathrm{Cu}_{76} \mathrm{Zn}_{16} \mathrm{Al}_{8}$ is obtained by induction melting of electrolytical $\mathrm{Cu}, \mathrm{Zn} 99,96 \%$ and $\mathrm{Al}$ 99,99\% in an evacuated chamber under a nitrogen rich atmosphere. Cylindrical ingots were obtained by in-situ pouring of the liquid alloy into molds. The preoriented single crystals of $\beta \mathrm{Cu}-\mathrm{Zn}-\mathrm{Al}$ were obtained by a modified Bridgman method. Homogeneization was carried out at $1123 \mathrm{~K}$ in evacuated quartz tubes subsequently quenched in water. The crystal is known to have a $\mathrm{DO}_{3}$ ordered $\beta$ phase at room temperature and an orthorombic $9 \mathrm{R}$ structure at low temperature. The $\mathrm{Ms}$ martensitic transformation temperature $(\mathrm{Ms}=267 \mathrm{~K})$ was determined by Differential Scanning Calorimetry (DSC).

The white synchrotron beam characteristics are $4.10-^{2}<\lambda<40.10^{-2} \mathrm{~nm}$ with $\lambda_{\max }=1.6 .10^{-1} \mathrm{~nm}$ when the power of the DCI ring is $2.9 .10^{-10} \mathrm{~J}$ and the intensity $3.10^{-1}$

A. The cross section of the beam is circular with a diameter of $10^{-2} \mathrm{~m}$. The diffracted beams can be recorded using either a TV camera or films or nuclear plates. In the latter case the exposure time is the order of 1 to 5 seconds.

Experiments were made in a regulated $( \pm 0.2 \mathrm{~K})$ Peltier cell whose temperature can vary from $233 \mathrm{~K}$ to $323 \mathrm{~K}$. To avoid the water condensation, during the temperature variations, the cell is put inside a chamber with a primary vacuum of $10^{-1} \mathrm{~Pa}$. This cell is built so as to allow the incident and diffracted X-Ray beams crossing.

Due to the high energy of the incident X-Ray beam in situ and real time studies are possible. The experimental technique is such that, when the first nuclei of the low temperature phase are detected, the temperature is slowly decreased in steps of $0.2 \mathrm{~K}$. To follow the crystal structure evolution and the lath growth, topograms are recorded at each step on films or nuclear plates. The reverse transformation is studied in a same manner as the direct one.

\section{3.- Results}

The martensitic lath interaction with the parent matrix is summed up in figure 1 . Figure 1a, 1b, .. 1h are the photographic enlargements of the $11 \overline{3}$ diffraction spots recorded during the sample cooling.

Figure 1a shows the initial crystalline state. The substructure characteristic of a single crystal, obtained by pulling technique, is clearly displayed : the linear black contrasts are due to the subboundaries. The white beam nature of the incident X-Ray beam allows to record the diffraction of each subgrain on the same diagram.

Figure $1 \mathrm{~b}$ shows the crystalline state of the same crystal after one complete $ß \rightleftarrows 9 R$ cycle. We note new "punctual" white-black contrasts due to crystalline defects resulting from the transformation.

Figure 1c. After the first cycle the crystal has been partially transformed so that only a single martensitic lath progresses. This one out of reflexion in the $B$ diffraction spot appears in white contrast and is noted " $A$ ". The diffraction spot " $B$ " of the $9 R$ martensite lath appears in black elsewhere. 


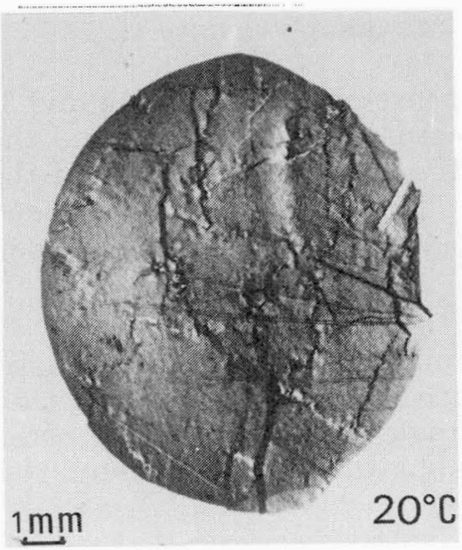

a

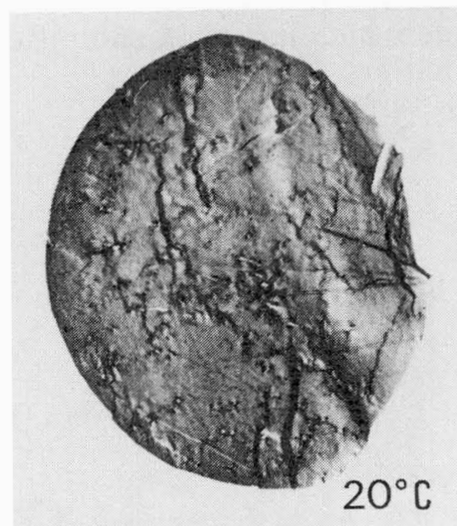

d

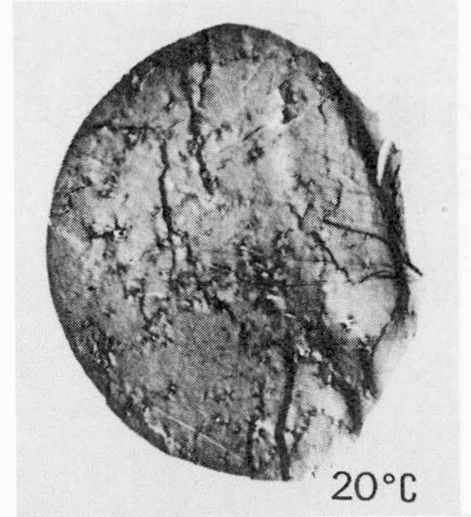

g

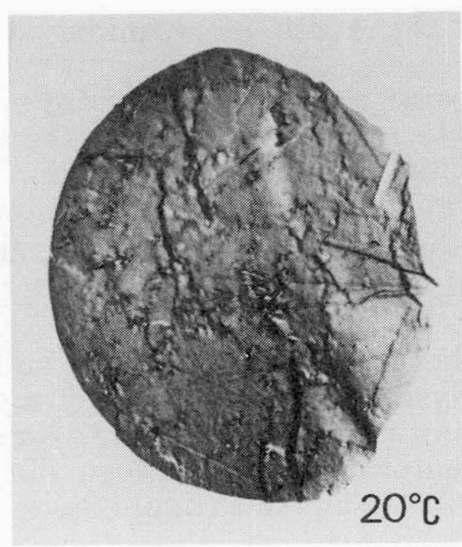

b

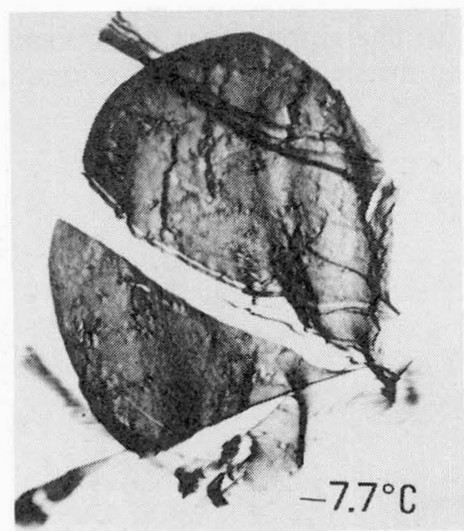

e

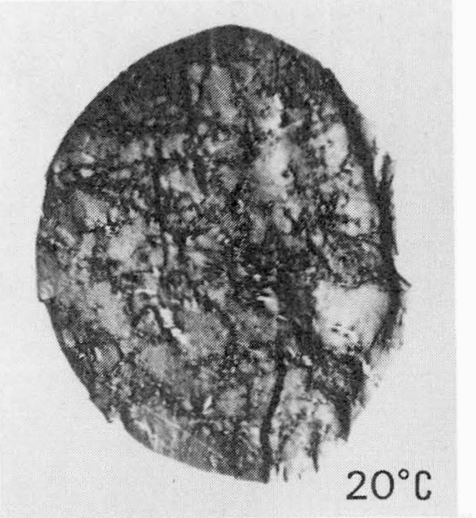

h

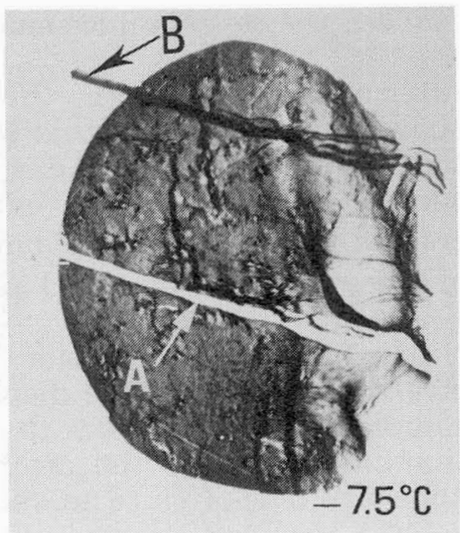

c

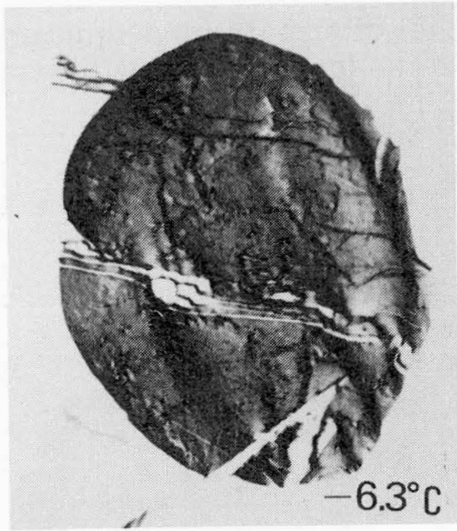

f

Figure 1

Photographic enlargement of the $11 \overline{3}$ diffraction spot given by a CuZnAl crystal submitted to partial and complete $\beta \vec{k} 9 \mathrm{R}$ cycles 
Figure 1d accounts for the $B$ crystalline state after a temperature increase which has induced the reverse movement of the lath, its shrinking and its disappearance.

After a new partial cycle the same plate shaped variant appears again. Figure 1e and $1 f$ show two positions of this plate respectively during the temperature decrease and the temperature increase. After total reverse transformation the $\beta$ crystalline state of the sample is presented in figure $1 \mathrm{~g}$. At this stage no new "punctual" defect has been created. If the crystal is submitted to a new total $B \rightleftarrows 9 R$ transformation the multiplication of these defects is observed in figure $1 \mathrm{~h}$.

During the transformation the crystalline lattice is submitted to long range elastic strains. These ones are displayed in the topograms of figure 2 obtained from an other sample. Figure $2 a$ shows the initial crystalline state of a single crystal. It can be noted that, according to the disorientation sense between the subgrains the subboundaries appear in black (figure $1 \mathrm{a}$ and $2 \mathrm{a}$ ) or in white (figure 2a).

The parent phase shape deformation, during the transformation, is displayed in the upper part of the figure $2 \mathrm{~b}$. After coming back in the high temperature phase (figure 2c) the crystalline state is comparable to the initial one (subboundary positions) but we note that aftermath have appeared in the upper part of the sample which has been submitted to the long range elastic strains during the partial transformation.

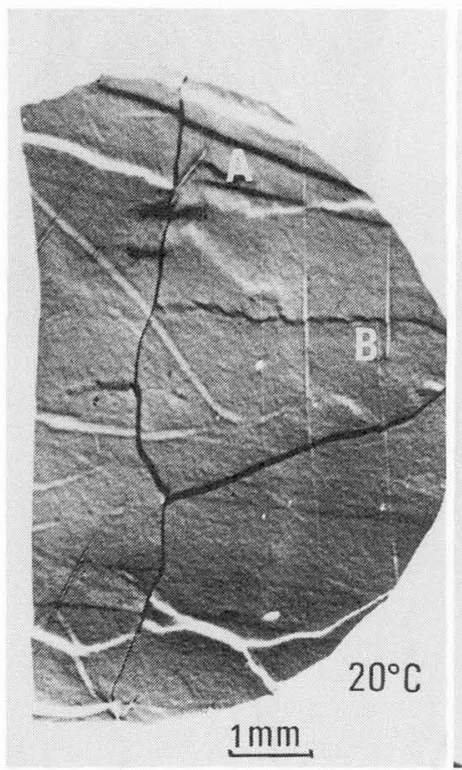

a

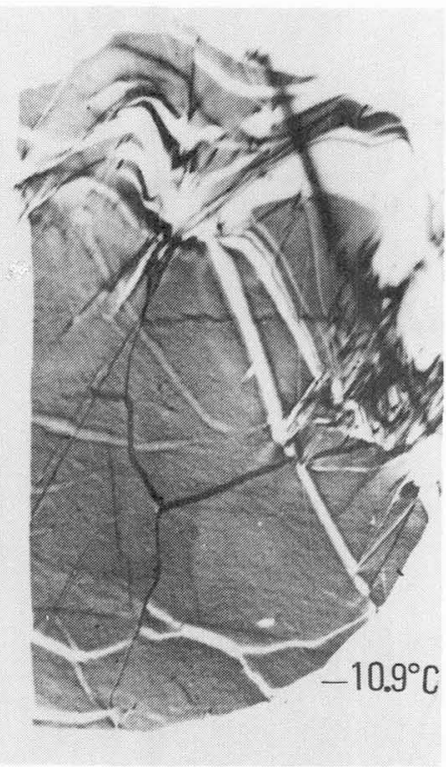

$\mathrm{b}$

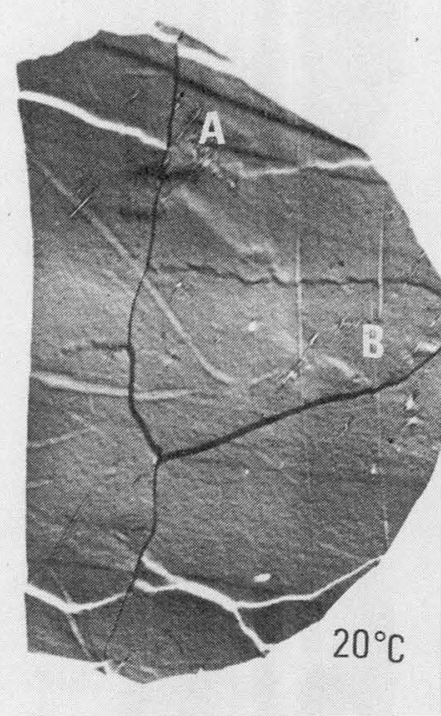

c

Fig. 2

CuZnAl crystal submitted to a partial $\beta \rightleftarrows 9 R$ cycle. The X-Ray topograms show the long range elastic strains ( $A$ and $B$ ) in the parent phase during the transformation and the aftermath ( $A$ and $B$ ) after coming back in the $B$ phase. 


\section{4.- Discussion}

The thermoelastic nature of this martensitic transformation is well illustrated by the topograms in figures 1 or 2. The macroscopic crystal structure is preserved after a partial or a total cycle and even after several cycles. The subboundaries appeared at the same place and the subgrain disorientations are conserved.

After a total cycle we have noted the apparition of "punctual" crystalline defects in the $B$ phase. We have tried to determine if these defects result from the interaction of the martensitic lathes with the crystalline lattice or if they were the consequence of the lath interactions between themselves or with the crystal defects. The experiments show, to the $\mathrm{X}$-Ray topography resolution, that when only a single martensitic lath grows or decreases it does not interact with the crystalline lattice and during its backward movement it does not leave defects (figure 1c to $1 \mathrm{~g}$ ). Nevertheless, if the crystal is submitted to a new complete transformation cycle we state again a multiplication of the "punctual" defects (figure 1h). A total transformation being multivariant we think that the observed aftermath are the consequences of the mutual variant interactions. This hypothesis is also confirmed by the topograms in figure 2.

\section{5.- Conclusion}

The Synchrotron White Beam X-Ray Topography is a well adapted method to study in situ and in real time the thermoelastic transformations. We have shown that after a total transformation cycle numerous "punctual" crystalline defects were observed which were not due to the lath interaction with the parent phase but could be due to their mutual interactions. Otherwise this method is also well adapted to visualize the large elastic strains.

\section{References}

11/ WARLIMONT, H. and DELAEY, L., Martensitic transformation in copper, silver and gold based alloys. Pergamon, Oxford, (1974)

/2/ RIOS-JARA, D., ESNOUF, C. and GUENIN, G., Phys. Stat. Sol. A87 (1985\} 187

/3/ MAI, C., GUENIN, G., MORIN, M., LIVET, F. and GOBIN, P.F., Mat. Sci. and Eng., 45 (1980) 217

14/ GASTALDI, J. and JOURDAN, C., Phys. Stat. Sol., 49 (1978) 523

/5/ GRANGE, G., GASTALDI, J. and JOURDAN, C., J. Cryst. Growth, 104 (1990) 851

/6/ JOURDAN, C., GASTALDI, J. and GRANGE, G., Acta Met., 36 (1988) 2979 